
\title{
Complications of Prophylactic Intercostal Tube Drainage - including Tension
Pneumothorax
}

\author{
Maj T S Burge \\ FRCS RAMC*
}

Department of Cardio-Thoracic Surgery, Royal Infirmary of Edinburgh EH3 9YW

SUMMARY: A case is presented of tension pneumothorax associated with intercostal tube drainage. Complications of intercostal tube drainage are reviewed.

\section{Introduction}

Intercostal tube drainage (ICD) has been advocated as treatment for air or fluid within the chest since at least 1876 (1). As the procedure has been found to have a low morbidity - c. $1 \%(2)$ - its prophylactic use has been advocated under trauma programs such as Advanced Trauma Life Support (ATLS) (3). Many possible complications are recorded $(4,5,6)$, some of these are listed in Table 1. The technique of open, blunt insertion, rather than using a sharp trochar, is held to minimise but not abolish these complications (2). Tension pneumothorax has not previously been reported as a complication.

\section{Table 1}

\section{Complications of ICDs (not exhaustive)}

1. Damage to intercostal nerve, artery or vein.

2. Damage to internal mammary artery or vein.

3. Damage to intrathoracic or abdominal organs.

4. Persistent pneumothorax.

5. Emphysema; subcutaneous or mediastinal.

6. Pulmonary oedema.

7. Failure of lung to expand.

8. Recurrent pneumothorax on ICD removal.

9. Local wound problems.

10. Infection (empyema).

11. Intercostal myalgia.

12. Horner's syndrome.

13. Mechanical problems:

a. Incorrect position.

b. Dislodgement/disconnection.

c. Seal water flowing into chest.

d. Blockage.

e. Leak of seal water.

\section{Case Report}

A 65 year old male pedestrian was hit by a car. He was intubated prior to transfer to hospital as he was said to have a Glasgow Coma Scale (GCS) score of 3 .

On arrival he had poor air entry bilaterally and left chest wall crepitus. He was cardiovascularly stable and had a soft abdomen. His GCS score was 3 and he had

*Now Lothian Regional Plastic Surgery Unit, St John's Hospital at Howden, Livingston, West Lothian. unreactive pupils at the midpoint. The other major injury was an obvious fracture of the right femur.

Chest X-ray (CXR) showed fractures of the left fifth $\vec{\omega}$

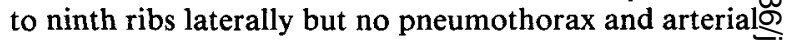
blood gases were satisfactory on $100 \%$ oxygen. Bronchoscopy showed a little blood in the right main? bronchus but all the major air passages were intact. Peritoneal lavage was negative.

The working diagnosis was of intracranial trauma as the main insult. It was felt that he might require positive pressure ventilation so bilateral ICDs as per ATLS guidelines were inserted. This was performed by an open technique, with a finger inserted into the chest agd without using a trochar. The left ICD had a large air leđik and the right was noted to not be swinging. CXR show 9 do a $20 \%$ left pneumothorax but otherwise everythinge seemed satisfactory. The patient then proceeded to $\vec{C}_{\overrightarrow{0}}$ (which was 'negative') by which time his GCS score hodd improved to c. 14.

At this point his condition suddenly deteriorate Systolic blood pressure fell from 140 to $40 \mathrm{~mm} \mathrm{Hg}, \mathrm{C} \mp$ rose from 7 to $15 \mathrm{~mm} \mathrm{Hg}$, inflation pressures rose to 35 -o $38 \mathrm{~cm} \mathrm{H}_{2} \mathrm{O}$ and the air leak into the left ICD became $\frac{\mathrm{Q}}{\mathrm{O}}$ much worse.

A further CXR revealed a right tension pneumothoraxo $\overrightarrow{\vec{O}}$ with the ICD indenting the lung outline. Repositioning of the right ICD led to a gush of air out followed by swinging for the first time. There was an immediate improvement in his clinical condition with a rise in systolic blood pressure and falls in CVP and inflation pressure to previous values.

At this point a previous medical history (PMH) of alcoholic cardiomyopathy and chronic obstructive $\frac{\mathcal{N}}{\vec{J}}$ airways disease became available.

He next developed persistent bleeding around the right ICD along with a right haemothorax. The origini appeared to be intercostal and the ICD was therefore resited one space down with a pressure being applied to the presumed bleeding point. This was achieved by inserting a large Foley catheter (connected to an un-o derwater seal) through the ICD hole, inflating the balloon with $50 \mathrm{ml}$ saline and hanging on it a weight (a). $500 \mathrm{ml}$ bag of saline). This controlled the bleeding bothn externally and internally (as evidenced by CXR andN ICD). 
The air leak on the left necessitated a double lumen tube to allow high frequency jet ventilation of the left lung while the right received 'normal' ventilation. Despite all these measures he was unable to maintain an adequate $\mathrm{pAO}_{2} \mathrm{~s}$ despite an $\mathrm{FiO}_{2}$ of 1.0 . In view of his PMH and poor prognosis active treatment was withdrawn and he died approximately 24 hours after admission.

Post mortem examination showed:

1. There was an area of contusion within the right oblique fissure, corresponding with the lung indentation seen on CXR.

2. There was a circular defect in the visceral pleura of the left lung, corresponding in size and site with the ICD. This may have been a ruptured emphysematous bulla.

\section{Discussion}

Complication of ICDs should be rare. Unfortunately this patient developed three problems which may have been due to this prophylactic measure:

1. Broncho-pleural fistula. This may have been due to ventilatory rupture of a pre-existing bulla, but there was no pneumothorax pre-ICD.

2. Haemorrhage from an intercostal vessel. This as the cause of bleeding on the right, was supported by the success of the remedy.

3. Tension pneumothorax. The exact source of the air was unclear. It is suggested however that it was unable to escape as the ICD was in the oblique fissure, blocking its holes and possibly creating a valve.

This case brings out two main points:

1. The insertion of ICDs, even by a blunt technique, is not without hazard. Particularly in the case of prophylactic use there must be a balanced assessment of the potential benefits against the potential dangers. "If it ain't broke, don't fix it".

2. The presence of an ICD - at least one that is not swinging - does not guarantee the absence of a tension pneumothorax.

\section{REFERENCES}

1. HeweTt F C. Thoracentesis: the plan of continuous aspiration. Br Med $J 1876 ; 1 ; 317$.

2. Millikan J S, Moore E E, Steiner E, Aragon G E, VAN WAY $C W$. Complications of tube thoracostomy for acute trauma. Am J Surg 1980; $140 ; 738-41$.

3. American College of Surgeons. Advanced trauma life support student manual 1988. Chicago: American College of Surgeons, 1989: 103.

4. American College of Surgeons. Advanced trauma life support student manual 1988. Chicago: American College of Surgeons, 1989: 108-9.

5. Fleishman J A, Bullock J D, Rosset J S, Beck R W. Iatrogenic Horner's syndrome secondary to chest tube thoracostomy. J Clin Neuro Opthalmol $1983 ; 3 ; 205-10$.
6. Trapnell D H, Thurston J G. Unilateral pulmonary oedema after pleural aspiration. Lancet 1970; 1: 1367.

\section{ADDENDUM \\ A technique for intercostal drain (ICD) insertion}

British Army Trauma Life Support (BATLS) teaching듬 provides the basis for a technique for ICD insertion that $\overline{\bar{\rho}}$ the author has found to be easy, safe and well tolerated.

The target is the fourth intercostal space between anterior and mid axillary lines. Here the interspaces are $e^{\infty}$ large, pectoralis major is not restricted as an accessory. respiratory muscle and the surgical assault is away from $\overrightarrow{ }$ the mediastinum and internal mammary artery. The ${ }_{\sigma}^{\omega}$ surface marking of the insertion point, in the supine patient with the arm fully abducted, is the posterior? border of the pectoralis major at the level of the nipple.

If local anaesthesia is required a large volume of dilute $\vec{\omega}$ agent (eg. $30 \mathrm{ml} 0.5 \%$ lignocaine) should be infiltrated into the proposed track paying particular attention to the skin and parietal pleura - the latter may be difficult. $\infty$ Presence of a pneumothorax will be confirmed by airo aspiration at this stage; aspiration of lung producessmall amounts of characteristic bright red, frothy bloo $\$$

The skin incision should be made transversely ope interspace caudally. It is better if it errs on the side of being too long, particularly in the obese. About 3-4 c్n seems to be right. A heavy silk vertical mattress suturegise inserted where the ICD will lie but left untied. It is useid? to seal the track when the ICD is removed. A further heavy silk suture is inserted and tied at one end of the incision. The ends are left long for fixing the ICD itself.

The track is created with a heavy clip bluntly dissecting\% OVER the fifth rib. On entering the pleural cavity thereo is usually a characteristic rush of air. The clip is $\overrightarrow{0}$ exchanged for a digit which is swept around through 3603 degrees to confirm the intrathoracic location and iden-? tify any adhesions.

The chosen ICD (large to avoid blockage eg. 283 French) is inserted, reusing the heavy clip to grip the tip and guide it into position. Ideally the drain should be directed apically for air and postero-basally for blood but with fenestrated drains this is not essential. While the use of a partially withdrawn trochar should be safe and? allow more precise direction of the ICD within the chest? the author prefers to keep large sharp objects out of the thoracic cavity except under direct vision.

The ICD is fixed with the prepositioned suture and connected to an underwater seal system. Fluid in the tube should swing with respiration and the seal should bubble.

The wound is closed snugly onto the ICD with inter-N rupted sutures, dressed and sealed with 'sleek'. 